\title{
The critical role of PPARa in the binary switch between life and death induced by endoplasmic reticulum stress
}

\author{
Ling Xu (1), Xiangying Zhang', Yuan Tian', Zihao Fan', Weihua Li', Mei Liu', Jianhua Hu', Zhongping Duan', \\ Ronghua $\operatorname{Jin}^{1}$ and Feng Ren ${ }^{1}$
}

\begin{abstract}
Endoplasmic reticulum stress (ER stress) just like a double-edged sword depending on different conditions in the development of multiple hepatic diseases. But the molecular mechanisms of functional conversion during ER stress have not been fully elucidated. In this study, we aim to illustrate the role of PPARa and the subtle mechanism in the functional conversion of ER stress. Tunicamycin (TM) and thapsigargin (TG), as ER stress inducers, were used to induce ER stress in AML12 cells. During the ER stress, QRT-PCR and immunoblotting was used to measure the expression levels of GRP78 and CHOP which show a gradually increasing trend, while PPARa and autophagy was significantly activated in the early stage but was inhibited in the late stage. Moreover, PPARa inhibition by siRNA promoted cell injury in the mild-ER stress and PPARa activation by WY-14643 reduced cell apoptosis in the serious ER stress. In the mild-ER stress with PPARa knocked down, activation of autophagy by rapamycin significantly improved cell survival, in the serious ER stress with PPARa activation, inhibition of autophagy by 3-MA aggravate cell injury. In addition, in the mild-ER stress with PPARa knocked down, CHOP knocked down by siRNA reduced cell apoptosis, in the serious ER stress activated PPARa, CHOP over-expression mediated by lentiviral vector contributed to serious cell injury. Furthermore, C57BL/6 mice was used to induce ER stress with TM intraperitoneal injection, PPARa and autophagy was upregulated in the mild-ER stress while downregulated in the serious ER stress, measured by qRT-PCR and immunoblotting, further confirmed the finding in vitro. Our results firstly demonstrated that PPARa is a key molecule in the functional conversion of ER stress: protective effects in the mild ER stress was mediated by PPARa-autophagy pathway and destructive effects in the serious ER stress was mediated by PPARa-CHOP pathway.
\end{abstract}

\section{Introduction}

In eukaryotic cells, the endoplasmic reticulum (ER) is responsible for multiple functions, such as protein folding and modification, lipid synthesis, and calcium storage and release. Conditions that may disturb ER homeostasis include surges in protein synthesis, energy deprivation, and imbalances of ER calcium levels. These conditions

\footnotetext{
Correspondence: Ronghua Jin (Jin_eagle@yahoo.com) or Feng Ren (renfeng7512@hotmail.com)

${ }^{1}$ Beijing Youan Hospital, Capital Medical University, No. 8, XitouTiao Road,

Youwai Street, Fengtai District, Beijing 100069, China

These authors contributed equally: Ling Xu, Xiangying Zhang

Edited by G.M. Fimia
}

lead to accumulation of unfolded or misfolded protein in the ER lumen, which is known as endoplasmic reticulum stress (ER stress). Hepatocytes are rich in ER content because of their high protein synthesizing capacity; therefore, ER stress-induced hepatocyte injury plays an important role in the pathogenesis of various liver diseases. Some studies have found that ER stress is involved in the progression of nonalcoholic fatty liver disease ${ }^{1}$. Further, rapid replication of hepatitis $C$ virus and accumulation of viral protein in the ER has been shown to trigger ER stress to promote hepatocytes damage ${ }^{2}$. Finally, arylating quinones have been used as clinical analgesics to

\section{(c) The Author(s) 2020}

(c) (i) Open Access This article is licensed under a Creative Commons Attribution 4.0 International License, which permits use, sharing, adaptation, distribution and reproduction c. in any medium or format, as long as you give appropriate credit to the original author(s) and the source, provide a link to the Creative Commons license, and indicate if changes were made. The images or other third party material in this article are included in the article's Creative Commons license, unless indicated otherwise in a credit line to the material. If material is not included in the article's Creative Commons license and your intended use is not permitted by statutory regulation or exceeds the permitted use, you will need to obtain permission directly from the copyright holder. To view a copy of this license, visit http://creativecommons.org/licenses/by/4.0/. 
induced liver injury, and they are closely associated with ER stress ${ }^{3}$.

In responding to ER stress, the unfolded protein response (UPR) signaling pathway is rapidly initiated in cells. The UPR is activated by three ER transmembrane proteins, protein kinase R-like ER kinase (PERK), inositol requiring 1 (IRE1), and activating transcription factor 6 (ATF6). Under physiological conditions, the peptides of three stress sensors in the ER lumen are bound to the chaperone glucose-regulated protein 78 (GRP78). When ER stress occurs, GRP78 disassociates with sensors and binds to misfolding proteins, alleviating the burden of the ER and leading to activation of the three sensors responsible for activating the UPR response. The UPR contributes to cell survival or death depending on the conditions of ER stress. In mild-ER stress, PERK is activated to phosphorylate eukaryotic initiation factor $2 \alpha$ (eIF2 $\alpha)$, which blocks intracellular protein synthesis by inhibiting mRNA translation to ease the ER load. IRE1 cleaves XBP1 mRNA to form XBP1s, then XBP1s work with ATF4-producing chaperones to promote protein folding and induce ER-associated degradation (ERAD). ATF6 is devoted to upregulating GRP78 and glucoseregulated protein 94 (GRP94). The UPR signaling cascade promotes the ability of the ER to restore homeostasis when encountering tolerable ER stress. In cases of persistent and severe ER stress, the UPR cannot resist stress and recover ER homeostasis. C/EBP homology protein (CHOP) upregulation, which is mediated by PERK, is considered a symbol of ER stress-induced apoptosis. IRE1 $\alpha$ induces proinflammatory and proapoptotic protein expression. When the UPR fails to attenuate stress in unresolvable ER stress, the apoptotic signaling pathway is activated. Therefore, ER stress functions as a doubleedged sword in various diseases. However, the molecular mechanism of switching the function of ER stress has not been elucidated.

Recently, ER stress has been proposed to be a switch between cell survival and death. Several studies have revealed molecular mechanisms that possibly participate in the shifting functional response to ER stress. BCL-2 family proteins, including proapoptotic and antiapoptotic members, act as specialized stress sentinels, and they activate molecular switches to transition between a prosurvival response and induction of apoptosis in response to irreversible stress ${ }^{4,5}$. In addition, researchers found that the loss of Klotho, a single-pass transmembrane protein that is expressed in multiple tissues ${ }^{6}$, is causally linked to ER stress-induced apoptosis and that overexpressing Klotho causes cells to be resistant to chemically induced ER stress ${ }^{7}$. Klotho could act as a molecular switch modulating ER stress signaling crosstalk between cell survival and death in the wound healing process ${ }^{8}$. Knockdown of E2F1, which belongs to the E2F family of transcription factors, increases the amount of cell death in response to ER stress. E2F1 plays a key role in the cell survival/death decision under ER stress ${ }^{9}$.

In our previous research, we demonstrated that in acute liver failure, peroxisome proliferator activated receptor alpha (PPAR $\alpha$ ) functions in ER stress-induced hepatocyte apoptosis ${ }^{10}$ and that PPAR $\alpha$ also plays an important role in the activation of autophagy ${ }^{11}$. Thus, we propose a hypothesis that PPAR $\alpha$ plays a key role in regulating the transition of cell fate towards survival or death in response to ER stress. Our results showed that PPAR $\alpha$ is a pivotal molecule that regulates the functional transition from protection to apoptosis in response to ER stress. The PPAR $\alpha$-autophagy pathway plays a protective role in promoting cell survival, and the PPAR $\alpha-C H O P$ pathway participates in severe ER stress-induced apoptosis.

\section{Materials and methods \\ Cell culture and treatment}

AML12 cells were purchased from American Type Culture Collection (ATCC, Manassas, VA, USA). The cells cultured in Dulbecco's modified Eagle's medium (DMEM, Thermo Fisher, Inc., Rockford, IL, USA) with $10 \%$ fetal bovine serum (FBS, Thermo Fisher, Inc.) and 1\% Pen-Strep (PS, Thermo Fisher, Inc.). Tunicamycin (TM, $10 \mu \mathrm{g} / \mathrm{ml}$, Sigma, St. Louis, MO, USA) or thapsigargin (TG, $1 \mu \mathrm{g} / \mathrm{ml}$, Sigma) were diluted in medium for the treatment of cells; they were used at different times or different doses to induce different severities of ER stress. The cells were incubated with TM $(20 \mu \mathrm{g} / \mathrm{ml})$ for $6 \mathrm{~h}$ to induce mild-ER stress and with TM $(20 \mu \mathrm{g} / \mathrm{ml})$ for $24 \mathrm{~h}$ to induce severe ER stress. A specific siRNA for PPAR $\alpha$ ( $5 \mathrm{nM}$, Jima, Suzhou, China) was transfected into cells $24 \mathrm{~h}$ before the 6-h TM $(20 \mu \mathrm{g} / \mathrm{ml})$ treatment, and the PPAR $\alpha$ activator Wy-14643 $(50 \mu \mathrm{M})$ was used to treat cells $2 \mathrm{~h}$ before the 24-h TM $(20 \mu \mathrm{g} / \mathrm{ml})$ treatment. A Lipofectamine 2000 kit (Invitrogen, ThermoFisher Scientific, Inc.) was used to transfect specific siRNAs according to the manufacturer's instruction. The autophagy inhibitor 3-methyladenine (3-MA, $5 \mathrm{mM}$, Sigma) was dosed into medium $1 \mathrm{~h}$ before TM administration, and the autophagy activator rapamycin $(10 \mu \mathrm{g} / \mathrm{ml}$, Sigma) was incubated with cells for $3 \mathrm{~h}$. The specific siRNA for CHOP ( $5 \mathrm{nM}$, Jima) was transfected into cells to silence the expression of CHOP before TM administration. The lentivirus overexpression vector for $\mathrm{CHOP}$ or a negative control (N.C.) vector was transferred into cells at the concentration of $1 \times 10^{6} / \mathrm{ml}$ for $48 \mathrm{~h}$ before TM administration. Polybrene reagent $(5 \mu \mathrm{g} / \mathrm{ml})$ was added to improve the transfection. The sequence of PPAR $\alpha$ siRNA was $5^{\prime}$ GGAGCUGCAAGAUUCAGAATT $3^{\prime}$, and the sequence of COHP siRNA was $5^{\prime}$ CUCUCCAGAUUCCAGUCAGdT dT $3^{\prime}$. The efficacy of the siRNA and lentivirus treatments was confirmed by immunoblotting. 


\section{Real-time reverse transcription-polymerase chain reaction (RT-PCR) assays}

TRIzol reagent was used to extract total RNA from cells and liver tissue. Total RNA was reverse-transcribed to produce cDNA with a Superscript ${ }^{\mathrm{TM}}$ III First-Strand Synthesis system (Invitrogen, Carlsbad, CA, USA). Reactions were then set up in $10 \mu \mathrm{l}$ total volumes, and they contained 1× SuperMix (Platinum SYBR Green qPCR kit; Invitrogen), cDNA $2 \mu \mathrm{l}$ and $0.5 \mu \mathrm{M}$ of each primer. The reaction conditions were as follows: $50^{\circ} \mathrm{C}$ for $2 \mathrm{~min}$ and $95^{\circ} \mathrm{C}$ for $5 \mathrm{~min}$, then 50 cycles of $95^{\circ} \mathrm{C}$ for $15 \mathrm{~s}$ and $60^{\circ} \mathrm{C}$ for $30 \mathrm{~s}$. The relative level of target mRNA expression was normalized to HPRT and was analyzed using the $2^{-\Delta \Delta C t}$ method.

\section{Immunoblotting assays}

RIPA buffer contains protease and phosphatase inhibiters was used to extract protein from AML12 cells and mice liver tissue. extract protein from AML12 cells and mouse liver tissue. Protein quantification was performed using a bicinchoninic acid (BCA) protein assay kit (Biomed, Beijing, China) according to the manufacturer's instructions. Protein was separated by sodium dodecyl sulfate-12\% polyacrylamide gel electrophoresis and was subsequently transferred overnight to PVDF membranes at $4{ }^{\circ} \mathrm{C}$. Monoclonal antibodies against PPAR $\alpha(1: 1000$, Abcam, Cambridge, MA, USA), GRP78, CHOP, Bcl-2related X-protein (Bax), B-cell lymphoma-extra-large (BCL-xL), caspase-3, cleaved-caspase-3 (1:1000, Cell Signaling Technology, Danvers, MA, USA) were diluted in TBST buffer with $5 \%$ skim milk, and then they were incubated at $4{ }^{\circ} \mathrm{C}$ overnight with the membranes. The next day, the membranes were washed in TBST buffer for $90 \mathrm{~min}$, and then they were incubated with horseradish peroxidase-conjugated secondary antibody (1:2000, Cell Signaling Technology) for $60 \mathrm{~min}$. An enhanced chemiluminescence system (Thermosphere, Inc.) was used to develop the band by exposure on an X-ray system.

\section{LDH and CCK-8 assays}

AML12 cells were plated at a density of $1 \times 10^{4}$ per well in a 96-well plate and were grown overnight. Cells were treated as previously described. Detection of the rate of cell death was performed with an LDH cytotoxicity assay kit (Beyotime, Shanghai, China) according to the manufacturer's instructions. A cell counting kit-8 assay (Beyotime) was used to detect cell viability.

\section{Flow cytometry}

An Annexin V-Phycoerythrin (PE)/7-Amino-Actinomycin (7-AAD) double staining assay (BD Bioscience, Franklin Lakes, NJ, USA) was used to detect apoptosis. AML12 cells were plated at a density of $1 \times 10^{6}$ cells/well in 6-well plates. After exposure to drug treatment, cells were resuspended in
$1 \times$ binding buffer and then were stained with $\mathrm{PE}$ and 7-AAD reagent for $15 \mathrm{~min}$ in the dark. The samples were analyzed by a FACScan flow cytometer (BD Bioscience), and the data were analyzed with FlowJo software.

\section{Animal treatments}

For this study, 8- to 10-week-old male C57BL/6 mice were purchased from Capital Medical University (CMU, Beijing, China). The mice were kept in the CMU animal facility and had ad libitum access to food and water. All experiments were performed strictly in accordance with the ethical guidelines of the Capital Medical University Animal Experimentation Committee and were in full compliance with the National Institutes of Health Guide for the Care and Use of Laboratory Animals.

The animals were randomly divided into specific groups. The appropriate sample size was used to ensure the credibility of results. One group of mice were intraperitoneally injected with TM (Sigma, St. Louis, MO, USA) at a concentration of $1.0 \mathrm{mg} / \mathrm{kg}$ over a range of time $(3,6,12$, and $24 \mathrm{~h})$, another was injected with the drug in various concentrations $(1.25,2.50,5.00$, and $10.00 \mathrm{mg} / \mathrm{kg})$ for $24 \mathrm{~h}$ to create different phases and severities of ER stress. After drug treatment, liver tissues were harvested, frozen and stored in a freezer $\left(-80^{\circ} \mathrm{C}\right)$, and serum samples were collected for further research.

\section{Liver function}

Liver function was assessed by analyzing the levels of alanine aminotransferase (ALT) and aspartate aminotransferase (AST). Blood collected from the mouse abdominal aorta was measured by a multiparametric analyzer (AU5400, Olympus, Japan) according to the automatic analysis program.

\section{Immunofluorescence assays}

Frozen sections or cells on slides were fixed in paraformaldehyde for $10 \mathrm{~min}$, washed in PBS buffer three times, and subsequently treated with Triton X-100 to permeabilize cell membranes. The sections were blocked in $10 \%$ goat serum and $3 \%$ BSA in PBS for 20 min. The following antibodies were incubated with the sections overnight at $4{ }^{\circ} \mathrm{C}$ : PPAR $\alpha$ (1:500, Abcam), CHOP and LC3 (1:500, Cell Signaling Technology). After washing in PBS, the slides were incubated with Alexa Fluor 488 goat antirabbit IgG or Alexa Fluor 568 goat anti-mouse IgG (1:250, Invitrogen, ThermoFisher Scientific, Inc.) for $30 \mathrm{~min}$ at room temperature. The nuclei were stained with DAPI $(1 \mu \mathrm{g} / \mathrm{ml}$, Abcam) for $5 \mathrm{~min}$. Finally, the images were analyzed by a Leica DM2500 fluorescence microscope.

\section{Statistical analysis}

The results are presented as the mean with the standard deviation. Statistical analysis was conducted using 
unpaired t-tests or one-way analysis of variance (ANOVA), and a $P$ value less than 0.05 is considered statistically significant.

\section{Results}

PPARa and autophagy-related genes expression profile at different phase of ER stress

AML12 cells were treated with TM at different times to induce different periods of ER stress. GRP78 and CHOP were used as markers to detect ER stress, and their levels gradually increased with ER stress, implying that the stress worsened. We observed that PPAR $\alpha$ mRNA was significantly upregulated at the earlier stage of ER stress, at $6 \mathrm{~h}$ of treatment; however, its expression was decreased at the later stage of ER stress (Fig. 1a). Furthermore, we examined the mRNA levels of LC3, ATG5, and p62, which were activated at the early stage of ER stress but decreased at the late stage of ER stress (Fig. 1a). Furthermore, another ER stress inducer, TG, was used to induce ER stress at different times. Interestingly, the mRNA levels of PPAR $\alpha$, LC3, ATG5, and p62 showed similar expression profiles following TM administration (Fig. 1b). Next, we analyzed the protein levels of PPAR $\alpha$ over different time periods of ER stress induced by TM. PPAR $\alpha$ was also elevated at the early stage of ER stress and decreased at the late stage of ER stress. LC3 and ATG5 were also increased at the early stage and then decreased. With autophagy activation at the early stage of ER stress, the protein level of p62 was decreased, but p62 accumulated at the late stage of ER stress (Fig. 1c, d). The same results were also shown following the administration of TG (Fig. 1e, f). Next, we detected the protein expression of PPAR $\alpha, \mathrm{CHOP}$ and LC3. The results showed that PPAR $\alpha$ was increased in the early stage of ER stress and decreased in the late stage of ER stress; CHOP expression increased with time, and LC3 was activated in the early stage of ER stress and decreased in the late stage of ER stress (Fig. S1). Therefore, PPAR $\alpha$ and autophagy were activated under mild-ER stress conditions and inhibited under serious ER stress conditions.

\section{PPARa and autophagy-related gene expression profiles after exposure to different levels of ER stress}

To further clarify the role of PPAR $\alpha$ and autophagyrelated genes after exposure to different levels of ER

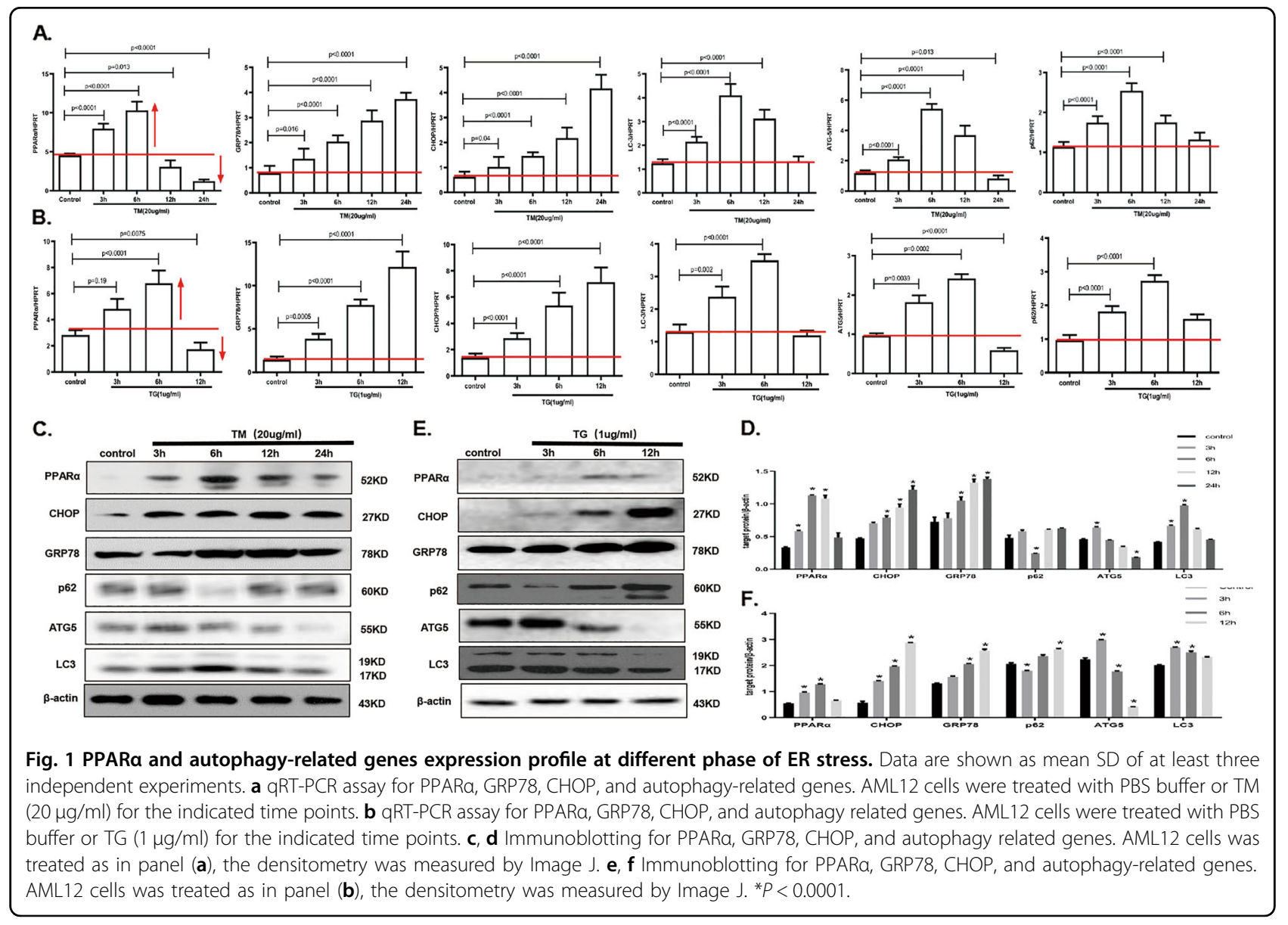




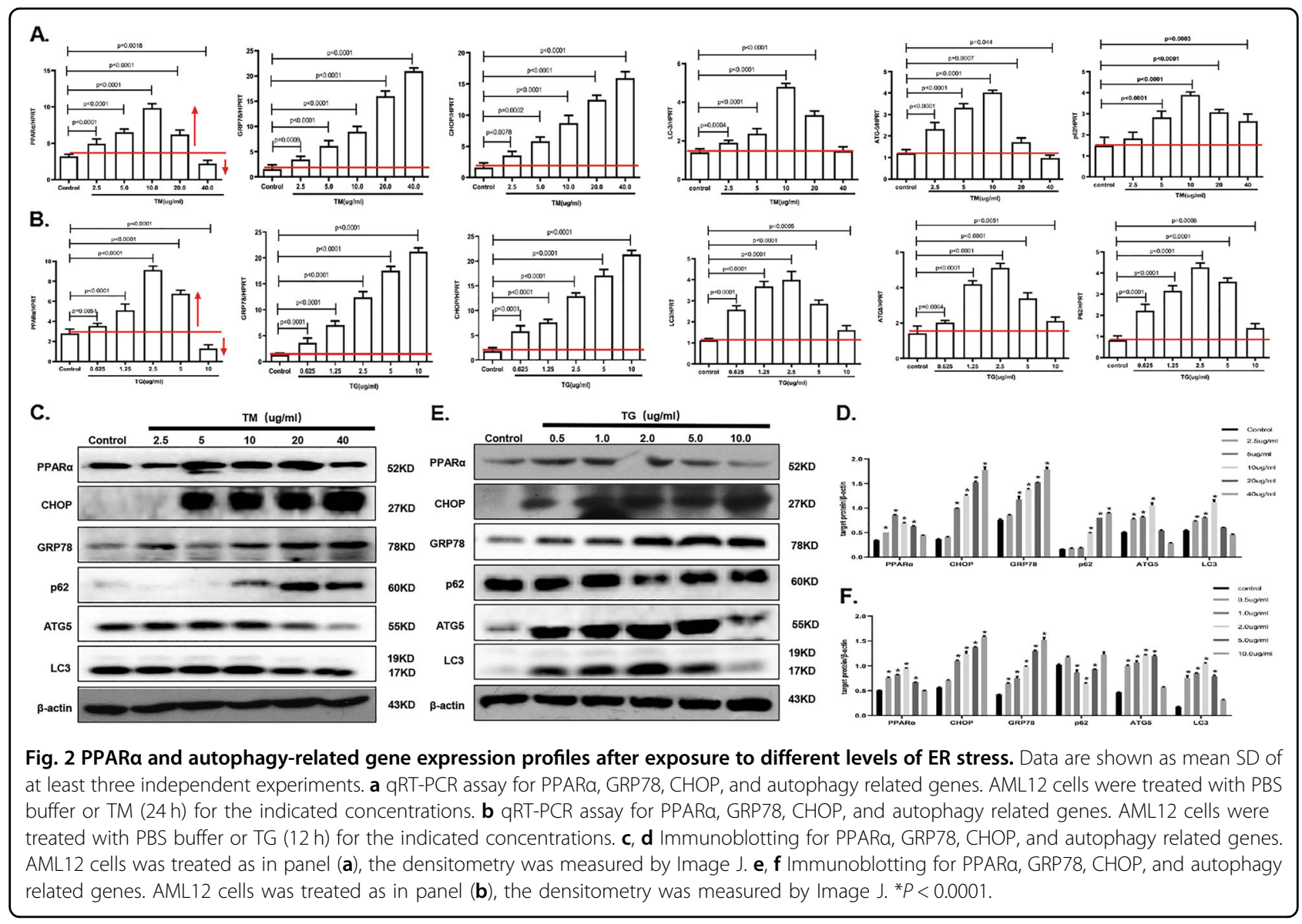

stress, we next investigated ER stress following treatment of cells with different concentrations of inducers. During low-dose TM induction, the mRNA levels of GRP78 and CHOP showed a slight increase compared with that of the control, suggesting that mild-ER stress was induced. During high-dose TM treatment, GRP78 and CHOP showed conspicuously high levels, suggesting that serious ER stress was induced. At the same time, PPAR $\alpha$ mRNA was elevated significantly in the low-dose treatment and gradually suppressed as the concentration increased (Fig. 2a). In addition, the mRNA levels of LC3, ATG5, and p62 were activated during low-dose drug exposure and inhibited during high-dose drug exposure (Fig. 2a). In addition, TG was used as another ER stress inducer, and the target gene mRNA expression profile was the same as that induced by TM (Fig. 2b). Next, we detected the protein level of target molecules. We found that PPAR $\alpha$ was increased under mild-ER stress and decreased under serious ER stress. In addition, ATG5 and LC3 protein levels were upregulated, while p62 decreased after lowdose TM exposure; however, ATG5 and LC3 decreased, while p62 accumulated after high-dose TM exposure (Fig. 2c, d). Following TG induction, we obtained the same results as those obtained after TM administration
(Fig. 2e, f). Furthermore, we detected the expression of PPAR $\alpha$, CHOP, and LC3 with immunofluorescence, and the expression profile was consistent with previous findings (Fig. S2). Overall, during the transition from mild-ER stress to serious ER stress, we observed that PPAR $\alpha$ and autophagy were promoted under mild-ER stress and suppressed under serious ER stress.

\section{Regulation of PPARa decides the fate of cells fate after exposure to different levels of ER stress}

Next, we explored the role of PPAR $\alpha$ in response to different levels of ER stress. AML12 cells were treated with TM for $6 \mathrm{~h}$ to induce mild-ER stress and $24 \mathrm{~h}$ to induce serious ER stress. The siRNA was used to knockdown PPAR $\alpha$ in mild-ER stress conditions, we used WY14643 was to activated PPAR $\alpha$ and lentivirus vector to upregulate PPAR $\alpha$, respectively in serious ER stress conditions. The effect of PPAR $\alpha$ siRNA treatment was detected by immunoblotting (Fig. S5). Knockdown of PPAR $\alpha$ promoted cell injury under mild-ER stress conditions, and activation of PPAR $\alpha$ under serious ER stress conditions evidently increased cell survival. The cell death rate measured by LDH assay also proved the same results (Fig. 3a). Furthermore, we used flow cytometry and 


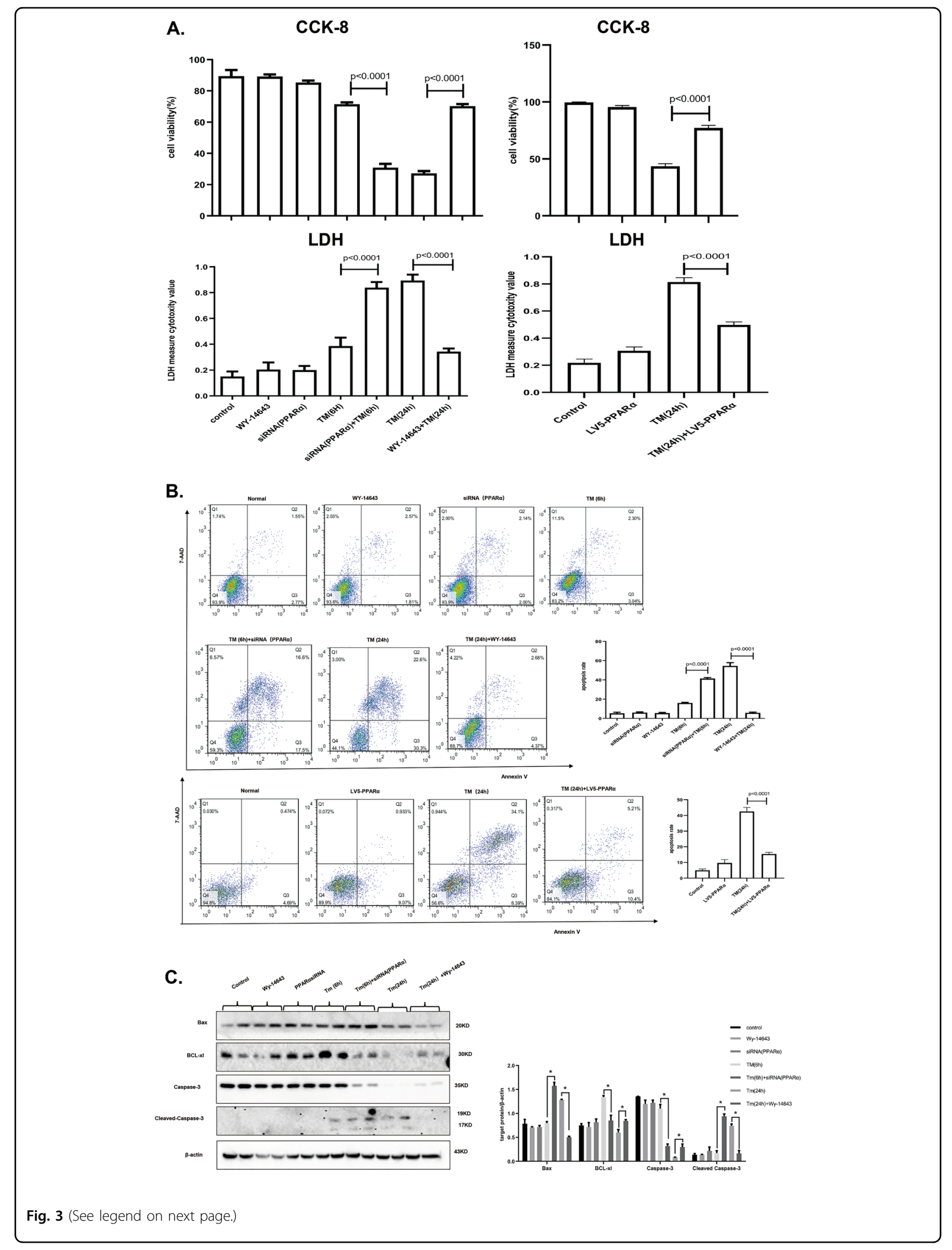


(see figure on previous page)

Fig. 3 Regulation of PPARa decides the fate of cells fate after exposure to different levels of ER stress. Data are shown as mean SD of at least three independent experiments. a CCK-8 and LDH assay for cell viability and cell death measurement. AML12 cells were treated with DMSO or WY$14643(50 \mu \mathrm{M})$ for $2 \mathrm{~h}$, then stimulated by TM $(20 \mu \mathrm{g} / \mathrm{ml})$ for $24 \mathrm{~h}$ and transferred with siRNA of PPARa $(5 \mathrm{nM})$ or control siRNA $(5 \mathrm{nM})$ for $24 \mathrm{~h}$, then treated with TM $(20 \mu \mathrm{g} / \mathrm{ml})$ for $6 \mathrm{~h}$. AML12 cells were transferred with LV5-PPARa at first, then stimulated by TM $(20 \mu \mathrm{g} / \mathrm{ml})$ for $24 \mathrm{~h}$. b Flow cytometry for cell apoptosis rate. AML12 cells was treated as in panel (a). The cells were recognized different populations in terms of their staining: the lower left quadrant was identified live cells, the lower right quadrant was identified early apoptosis cells, the upper left quadrant was identified necrotic cells, and the upper right quadrant was identified late apoptosis cells. The apoptosis rate was quantified by sum of early and late apoptosis. $\mathbf{c}$ Immunoblotting for Bax, BCL-XL, caspase-3, cleaved caspase-3. AML12 cells was treated as in panel (a). The densitometry was measured by Image $J$. ${ }^{*} P<0.0001$.

TUNEL assay, found that knocking down PPAR $\alpha$ under mild-ER stress conditions increased ER stress-induced apoptosis, while activating PPAR $\alpha$ under serious ER stress conditions significantly decreased ER stress-induced apoptosis (Figs. 3b and S6). Furthermore, we examined the protein levels of caspase-3, cleaved-caspase-3, Bax, and BCL-XL and found that cleaved-caspase- 3 and Bax were significantly upregulated, while BCL-XL was reduced under mild-ER stress conditions following PPAR $\alpha$ knockdown. Cleaved-caspase-3 and Bax were decreased, and BCL-XL was increased under serious ER stress conditions (Fig. 3c). Therefore, inhibition of PPAR $\alpha$ significantly aggravated cell injury under mild-ER stress conditions, while activation of PPAR $\alpha$ relieved serious ER stress-induced apoptosis.

Mild-ER stress mediates the ability of PPARa to restore cell homeostasis via autophagy activation, and serious ER stress inhibits the ability of PPARa to promote apoptosis via autophagy impairment

PPAR $\alpha$ was knocked down under mild-ER stress conditions, and ATG5 and LC3 protein expression was low, while p62 accumulated; these results indicated that the autophagy process was impaired. In serious ER stress conditions, where PPAR $\alpha$ was activated, ATG5 and LC3 protein expression was also activated, and p62 was decreased, indicating that autophagy was promoted (Fig. 4a). In conditions of mild-ER stress, which inhibited PPAR $\alpha$, rapamycin was used to activate autophagy, and the results showed that cell survival was significantly improved and that mortality and the cell apoptosis rate were decreased. In addition, under serious ER stress conditions, where PPAR $\alpha$ was activated, autophagy was blocked by 3-MA, leading to a decrease in the cell survival rate and a significant increase in the death and apoptosis rates (Fig. 4b, c). The inhibition of autophagy counteracts the protection of PPAR $\alpha$ activation, and autophagy activation abolishes PPAR $\alpha$ inhibition injury. Thus, the protective effect of PPAR $\alpha$ during mild-ER stress was mediated by the promotion of autophagy.

\section{Serious ER stress downregulates PPARa to promote apoptosis via CHOP activation, and mild-ER stress upregulates PPARa to restore homeostasis via CHOP inhibition}

We next examined the expression level of CHOP in cells with PPAR $\alpha$ knocked down in mild-ER stress conditions and in serious ER stress conditions where PPAR $\alpha$ was activated. The results show that $\mathrm{CHOP}$ was upregulated in the former group and downregulated in the latter group (Fig. 5a). Then, in the mild-ER stress conditions with knockdown of PPAR $\alpha, \mathrm{CHOP}$ was blocked by treatment with an siRNA, and there was an evident improvement in the cell viability rate and a decrease in the death rate and apoptosis rate. Under serious ER stress, in which PPAR $\alpha$ was activated, CHOP was overexpressed, resulting in a decline in the cell survival rate and an increase in the death rate and apoptosis rate (Fig. 5b, c). The effect of CHOP siRNA treatment was shown, and TM treatment for $24 \mathrm{~h}$ was used as a positive control. Lentivirus-mediated CHOP overexpression was also detected, and PPAR $\alpha$ expression was not affected by overexpression of CHOP (Fig. S5). This result shows that inhibition of $\mathrm{CHOP}$ could protect the cell from injury caused by mild-ER stress with inhibition of PPAR $\alpha$, and overexpression of CHOP could promote cell injury in serious ER stress, wherein PPAR $\alpha$ is activated.

\section{PPARa and autophagy-related gene expression profiles at} different times of TM-induced ER stress in a mouse model

To verify this in vitro phenomenon, we next explored the role of PPAR $\alpha$ in the functional conversion of ER stress in vivo. We first examined liver function by analyzing AST and ALT. With prolonged ER stress, the liver injury of animals worsened (Fig. 6a). Next, the mRNA of target genes was analyzed during times of ER stress. CHOP and GRP78 gradually increased, and PPAR $\alpha$ was upregulated in the early stage and were downregulated in the late stage. These results were consistent with those of the in vitro experiment. In addition, autophagy was activated at the early stage and suppressed at the late stage (Fig. 6b). Furthermore, the protein level of PPAR $\alpha$ showed 


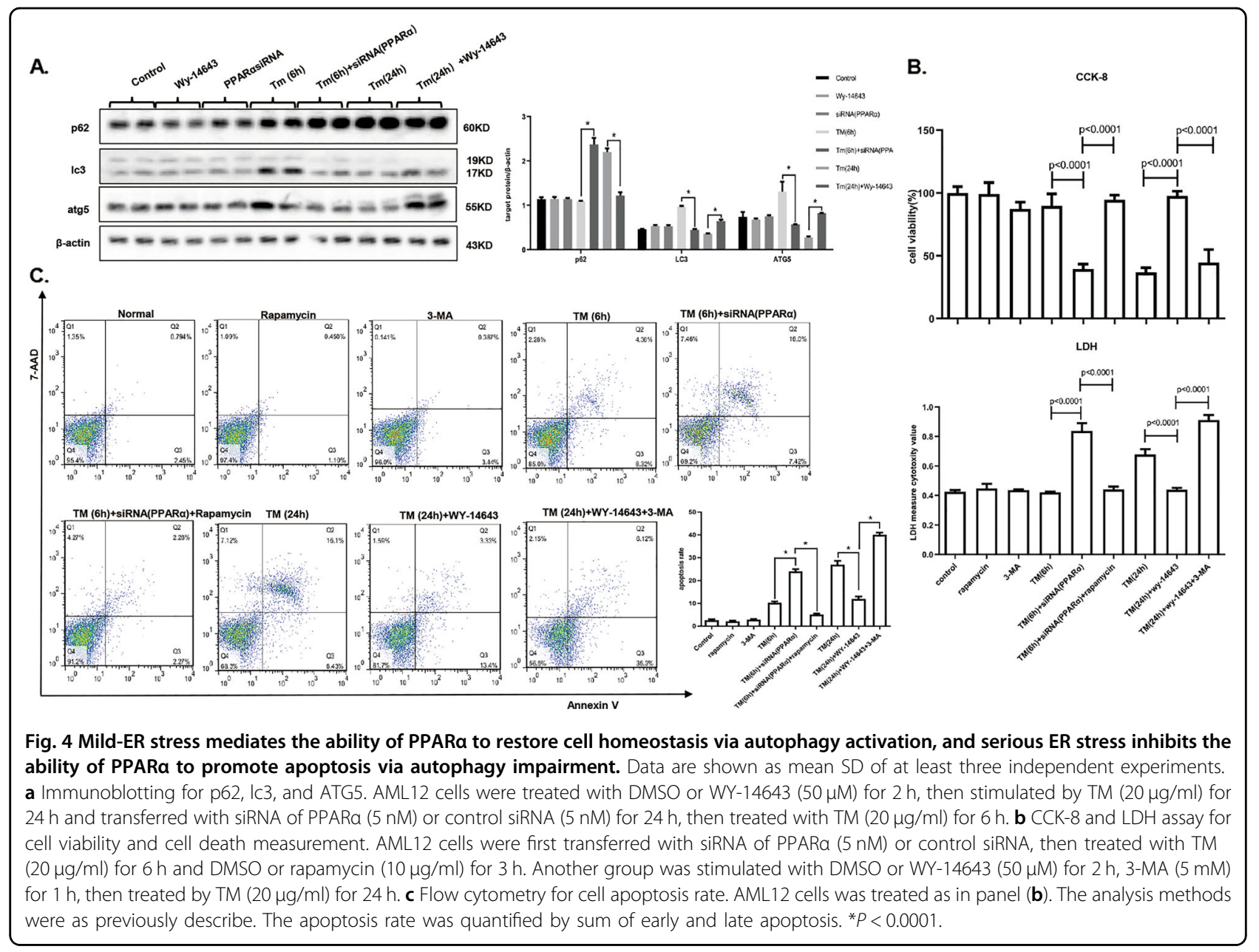

the same trends that were observed in the mRNA data. ATG5 and LC3 were upregulated, and p62 was decreased at an early stage, suggesting that autophagy was activated. ATG5, LC3 were decreased, and p62 was accumulated at a late stage, suggesting that autophagy was impaired (Fig. 6c). Immunofluorescence detection of PPAR $\alpha$, CHOP and LC3 also exhibited a phenomenon that was similar to what was observed in vitro (Fig. S3). As mice were exposed to different severities of ER stress, we saw that PPAR $\alpha$ and autophagy were activated under mild-ER stress and inhibited under serious ER stress.

PPARa and autophagy-related gene expression profiles at different doses of TM-induced ER stress in a mouse model

Then, different levels of ER stress were induced by treatment with different concentrations of TM. We first assessed the liver function of mice, which showed a gradual elevation of AST and ALT with increasing treatment concentrations (Fig. 7a). In addition, the mRNA levels of GRP78 and CHOP showed a slight increase under mildER stress conditions and a significant increase under serious ER stress conditions. However, the mRNA level of PPAR $\alpha$ was elevated under mild-ER stress and reduced under serious ER stress. Furthermore, autophagy-related genes were activated under mild-ER stress conditions and decreased under serious ER stress conditions (Fig. 7b). In addition, protein levels were analyzed, and PPAR $\alpha$ was at a high level under mild-ER stress conditions, but it was at lower levels under serious conditions. In addition, the autophagy factors ATG5 and LC3 were activated under mild-ER stress conditions and decreased under serious ER stress conditions. In addition, p62 degradation occurred under mild-ER stress conditions and accumulation occurred under serious ER stress conditions (Fig. 7c). In addition, with the increasing expression level of CHOP, PPAR $\alpha$ shows an increase in mild-ER stress conditions and a decrease in serious ER stress conditions. LC3 was also activated under mild-ER stress conditions and suppressed under serious ER stress conditions (Fig. S4). Thus, PPAR $\alpha$ and autophagy were activated under mild-ER stress conditions and inhibited under serious ER stress conditions. 


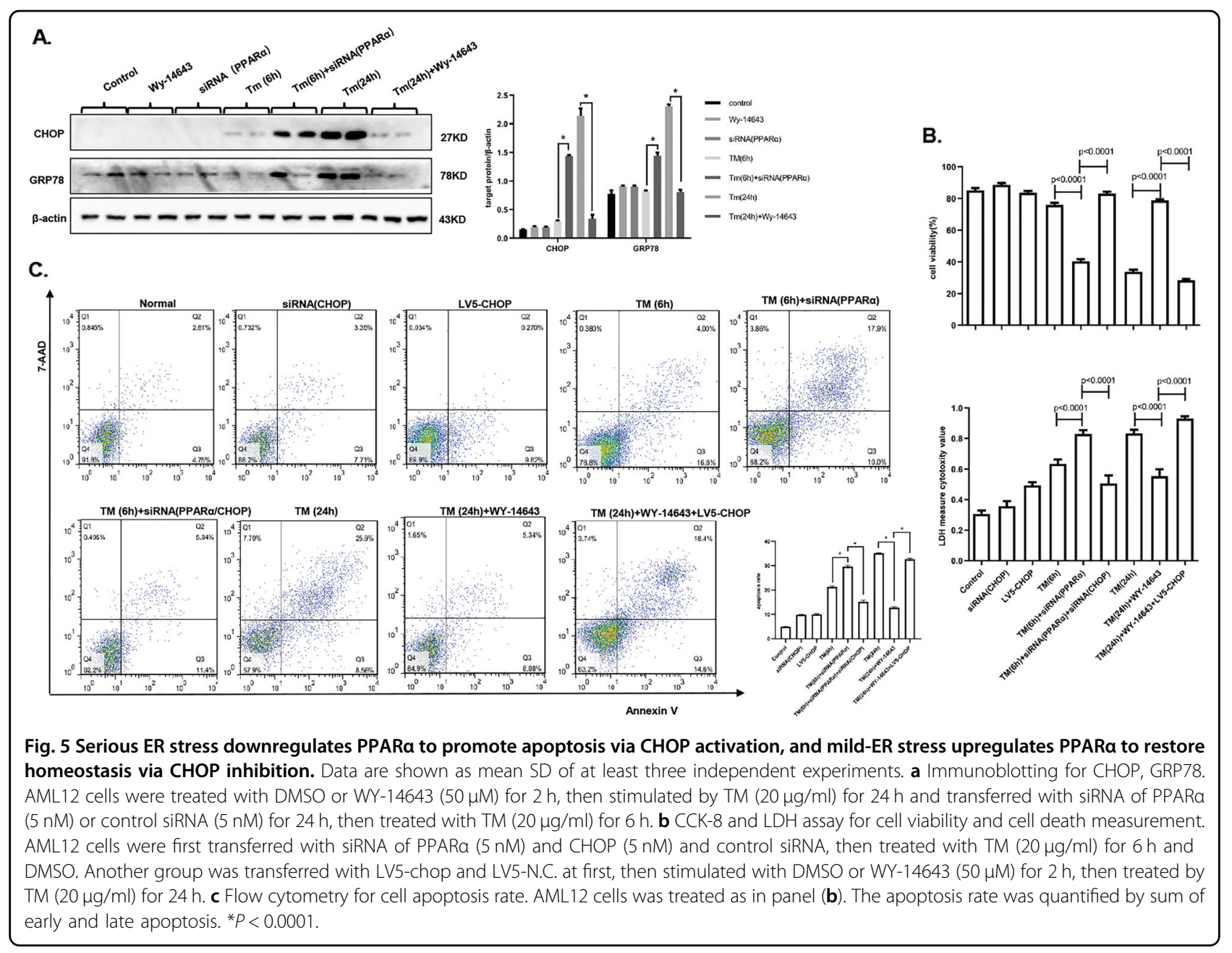

\section{Discussion}

Understanding the mechanisms of ER stress function conversion could enable the development of different strategies to control the function of ER stress at different stages of disease, thus providing a new strategy for disease prevention and treatment. In this study, with the transition of ER stress from mild to severe, PPAR $\alpha$ was upregulated under mild-ER stress conditions but downregulated under serious ER stress conditions. Furthermore, we demonstrated that autophagy was activated under mild-ER stress conditions and inhibited under serious ER stress conditions. Severe ER stress directly led to impaired autophagy. ATG5 and LC3 expression decreased, and impaired autophagy attenuated p62 degradation, leading to its accumulation ${ }^{12}$. In mild-ER stress conditions, inhibition of PPAR $\alpha$ resulted in a significant amount of apoptosis; anti-apoptosis genes were decreased, and pro-apoptosis genes were activated. Furthermore, the treatment led to autophagy blockade and CHOP overexpression. In serious ER stress conditions, activation of PPAR $\alpha$ played a protective role in decreasing cell apoptosis. In addition, autophagy was activated, and CHOP was downregulated in this process. With the inhibition of PPAR $\alpha$ in mild-ER stress conditions, knockdown of CHOP played a significant protective role in reducing apoptosis and promoting cell survival. With the activation of PPAR $\alpha$ in serious ER stress conditions, CHOP decreased cell viability. In addition, in mild-ER stress conditions, which decreased PPAR $\alpha$, promoting autophagy ameliorated cell damage; in serious ER stress conditions, which activated PPAR $\alpha$, suppressing autophagy could exacerbate cell injury. Finally, PPAR $\alpha$ expression profile in different stress conditions was demonstrated in vivo. This study reveals that PPAR $\alpha$ is a switch in ER stress function conversion; PPAR $\alpha$ activation promotes autophagy to relieve ER stress and promote cell survival, and PPAR $\alpha$ inhibition upregulates $\mathrm{CHOP}$ to promote apoptosis.

PPAR $\alpha$, the first member of a subfamily of nuclear receptors (PPARs), also includes PPAR $\beta$ and PPAR $\gamma$. PPARs form a heterodimer with retinoid $X$ receptor (RXR) to recognize specific DNA sequences and induce 


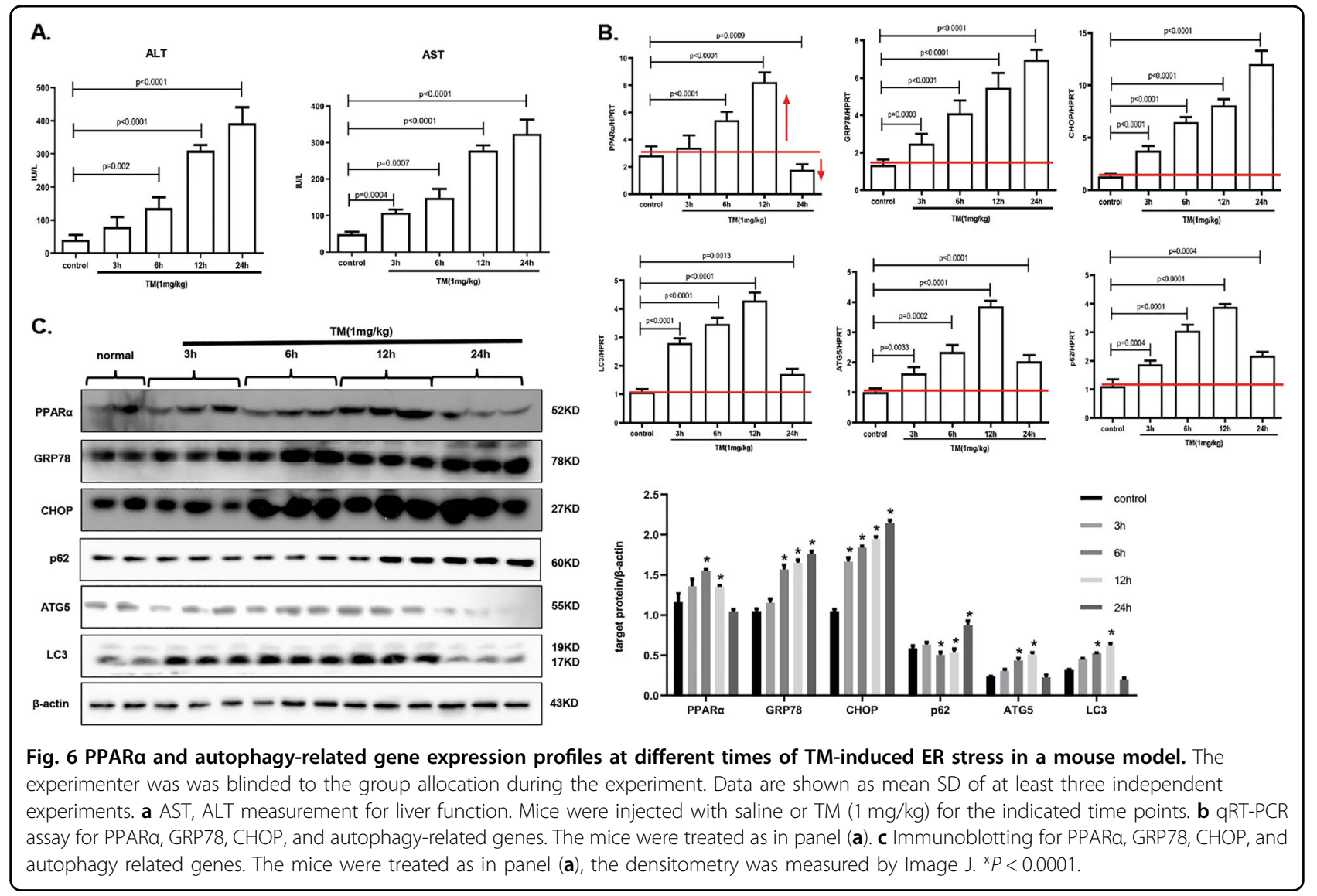

the expression of target genes ${ }^{13}$. PPAR $\alpha$ is involved in a series of physiological processes, including mitochondrial fatty acid oxidation, catabolism, the inflammatory response, and the stress response. Recent studies have identified a possible role of PPAR $\alpha$ in the ER stress response. Research has shown that activated PPAR $\alpha$ exerts a protective effect by inhibiting myocardial ischemia/reperfusion injury-induced ER stress ${ }^{14}$. Further, PPAR $\alpha$ can suppress the transcription of ATF2 to reduce ER stress-induced apoptosis in adipocytes ${ }^{15}$, and activation of PPAR $\alpha$ can protect against increased ER stress to ameliorate hepatic insulin resistance as well as lipid accumulation $^{16}$. Overall, multiple studies have shown that PPAR $\alpha$ activation plays a protective role in regulating ER stress during disease development. Our data further demonstrated the subtle regulation of PPAR $\alpha$ activation in mild-ER stress conditions promotes cell survival, and PPAR $\alpha$ inhibition in serious ER stress conditions promotes apoptosis.

Autophagy is an adaptive cellular process that plays a "housekeeping" role in physiological conditions through lysosomal-dependent pathways that digest intracellular organelles and remove damaged cellular components ${ }^{17}$. In response to ER stress, autophagy is activated to remove ubiquitous unfolded/misfolded proteins and reduce ER stress $^{18,19}$. However, there are studies showing that excessive autophagy can lead to aggravated cell injury ${ }^{20}$. The effects of autophagy induced by ER stress have a dual role, and they depend on the extent of ER stress ${ }^{21}$. Our data demonstrate that autophagy is activated in mild-ER stress conditions and impaired in serious ER stress conditions, providing a potential mechanism for the dual role of autophagy in ER stress. As a transcription factor, PPAR $\alpha$ regulates numerous target genes that participate in cell adaptive procedures in response to stress. Studies have shown that the nuclear receptors PPAR $\alpha$ regulate autophagy by controlling transcription of genes involved in autophagy pathways ${ }^{22}$; autophagy activation mediated by the upregulation of PPAR $\alpha$ could relieve acute liver failure in mice ${ }^{23}$. In this study, our results reveal that PPAR $\alpha$ activation in mild-ER stress conditions promotes restoration of homeostasis via promotion of autophagy.

When excessive ER stress was found to overwhelm the UPR compensation capacity, apoptosis was initiated. $\mathrm{CHOP}$ was upregulated and accumulated in the nucleus during apoptosis induced by ER stress ${ }^{24}$. Our previous research has shown that PPAR $\alpha$ inhibition mediates $\mathrm{CHOP}$ to promote the inflammatory response in acute liver failure ${ }^{11}$. Additionally, in NAFLD development, the progressive suppression of PPAR $\alpha$ was associated with the 


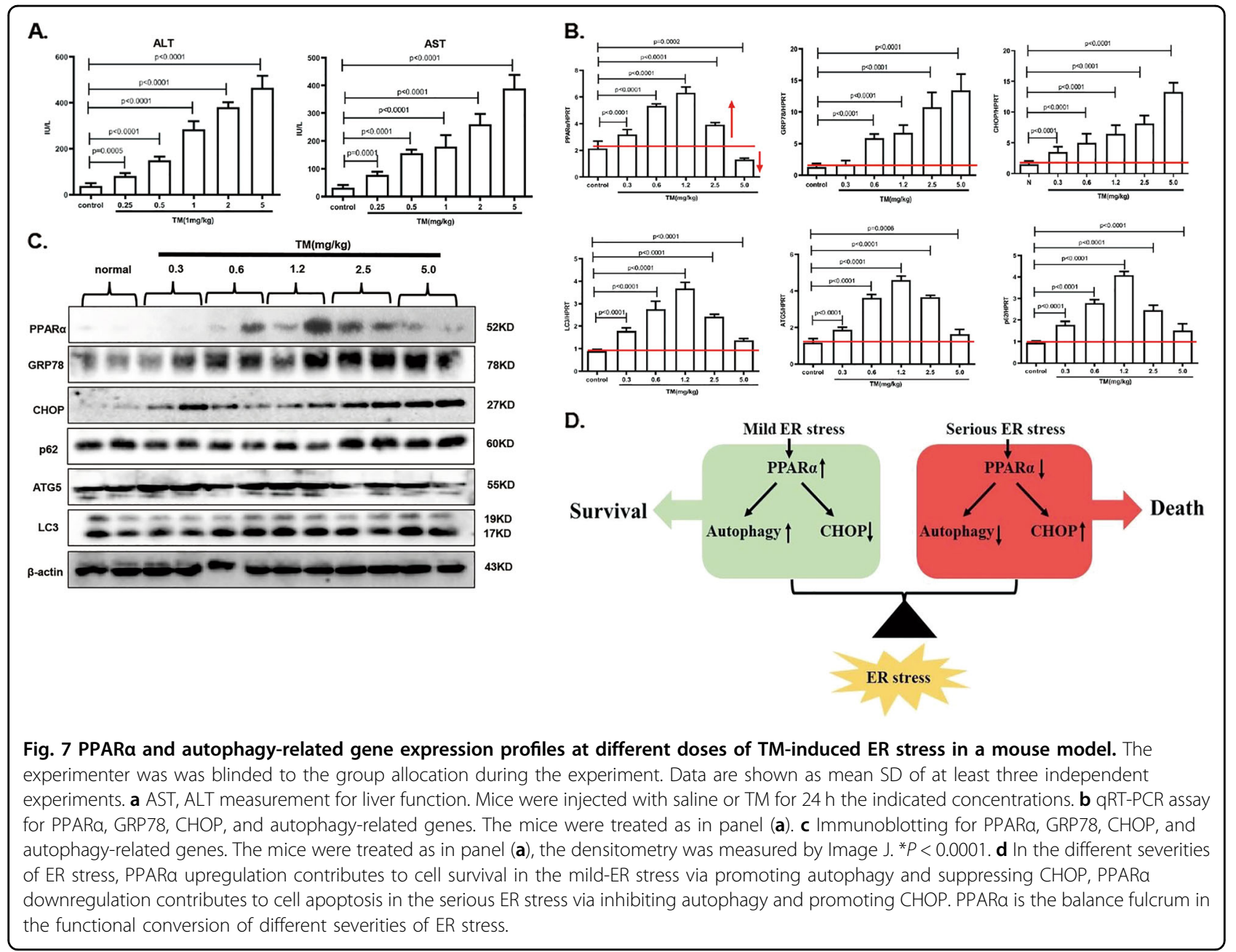

upregulation of $\mathrm{CHOP}^{25}$. Our results show that PPAR $\alpha$ inhibition induced apoptosis in serious ER stress via CHOP activation.

In summary, we verified that PPAR $\alpha$ is a key mediator in the functional conversion of ER stress response. ER stress is known to contribute to nearly all forms of liver diseases, so it is important to recognize the function of ER stress; further studies are still needed to explore the more precise mechanism of ER stress response. Distinct regulation of ER stress function based on the different stages of disease development new insight into clinical treatment.

\section{Acknowledgements}

This study was supported by grants from the National natural science foundation of China (81770611), Demonstrating application and research of clinical diagnosis and treatment technology in Beijing (Z191100006619096 and Z191100006619097); key projects of Beijing Municipal Education Commission's Science and Technology Plan (KZ202010025035), Beijing Municipal

Administration of Hospitals Clinical Medicine Development of Special Funding Support (XMLX201830), Key Medical Major of Beijing Sailing Plan (ZYLX201819) and the National Science and Technology Key Project on "Major Infectious Diseases such as HIV/AIDS, Viral Hepatitis Prevention and Treatment" (2018ZX10301407-005-002 and 2018ZX10302205-004-004).

\section{Author contributions}

F.R. and R.J. designed the experiments. L.X. and X.Z. performed the experiments and wrote the paper. Y.T., W.L., and Z.F. supervised the pathological observations. M.L. prepared the samples and collected the data. Z.D. and J.H. performed the statistical analyses. All authors have read and approved the submission of the paper.

\section{Conflict of interest}

The authors declare that they have no conflict of interest.

\section{Publisher's note}

Springer Nature remains neutral with regard to jurisdictional claims in published maps and institutional affiliations.

Supplementary Information accompanies this paper at (https://doi.org/ 10.1038/s41419-020-02811-4).

Received: 30 January 2020 Revised: 13 July 2020 Accepted: 14 July 2020 Published online: 11 August 2020 


\section{References}

1. Song, M. J. \& Malhi, H. The unfolded protein response and hepatic lipid metabolism in non alcoholic fatty liver disease. Pharmacol. Ther. 203, 107401 (2019).

2. Chusri, P. et al. HCV induces transforming growth factor beta1 through activation of endoplasmic reticulum stress and the unfolded protein response. Sci. Rep. 6, 22487 (2016).

3. Wang, $X$. et al. Mechanism of arylating quinone toxicity involving Michae adduct formation and induction of endoplasmic reticulum stress. Proc. Nat Acad. Sci. USA 103, 3604-3609 (2006).

4. Pihan, P., Carreras-Sureda, A. \& Hetz, C. BCL-2 family: integrating stress responses at the ER to control cell demise. Cell Death Differ. 24, 1478-87. (2017).

5. Rojas-Rivera, D., Caballero, B., Zamorano, S., Lisbona, F. \& Hetz, C. Alternative functions of the $\mathrm{BCL}-2$ protein family at the endoplasmic reticulum. $A d v$. Exp. Med. Biol. 687, 33-47 (2010).

6. Boksha, I. S., Prokhorova, T. A., Savushkina, O. K. \& Tereshkina, E. B. Klotho protein: its role in aging and central nervous system pathology. Biochem. Biokhimiia 82, 990-1005 (2017).

7. Banerjee, S. et al. Klotho ameliorates chemically induced endoplasmic reticulum (ER) stress signaling. Cell. Physiol. Biochem. 31, 659-672 (2013).

8. Mytych, J., Solek, P. \& Koziorowski, M. Klotho modulates ER-mediated signaling crosstalk between prosurvival autophagy and apoptotic cell death during LPS challenge. Apoptosis 24, 95-107 (2019).

9. Pagliarini, V. et al. Downregulation of E2F1 during ER stress is required to induce apoptosis. J. Cell Sci. 128, 1166-1179 (2015).

10. Zhang, L. et al. Peroxisome proliferator-activated receptor alpha acts as a mediator of endoplasmic reticulum stress-induced hepatocyte apoptosis in acute liver failure. Dis. Models Mech. 9, 799-809 (2016).

11. Zhang, $\mathrm{X}$. et al. Peroxisome proliferator-activated receptor alpha mediates C/ EBP homologous protein to protect mice from acute liver failure. Inflamm. Res. 66, 813-22. (2017)

12. Zhang, $\mathrm{H}$. et al. DEAD box protein 5 inhibits liver tumorigenesis by stimulating autophagy via interaction with p62/SQSTM1. Hepatology 69, 1046-2063 (2019).

13. Kersten, S. Integrated physiology and systems biology of PPARalpha. Mol. Metab. 3, 354-371 (2014).
14. Yuan, J. et al. PPARalpha activation alleviates damage to the cytoskeleton during acute myocardial ischemia/reperfusion in rats. Mol. Med. Rep. 17, 7218-26. (2018).

15. Liu, Z. et al. Adiponectin reduces ER stress-induced apoptosis through PPARalpha transcriptional regulation of ATF2 in mouse adipose. Cell Death Dis. 7. e2487 (2016)

16. Chan, S. M. et al. Activation of PPARalpha ameliorates hepatic insulin resistance and steatosis in high fructose-fed mice despite increased endoplasmic reticulum stress. Diabetes 62, 2095-2105 (2013).

17. Ravanan, P., Srikumar, I. F. \& Talwar, P. Autophagy: the spotlight for cellular stress responses. Life Sci. 188, 53-67 (2017).

18. Feng, J. et al. Autophagy activated via GRP78 to alleviate endoplasmic reticulum stress for cell survival in blue light-mediated damage of A2E-laden RPEs. BMC Ophthalmol. 19, 249 (2019).

19. Cheng, Y. C., Chang, J. M., Chen, C. A. \& Chen, H. C. Autophagy modulates endoplasmic reticulum stress-induced cell death in podocytes: a protective role. Exp. Biol. Med. 240, 467-476 (2015).

20. Ciechomska, I. A., Gabrusiewicz, K., Szczepankiewicz, A. A. \& Kaminska, B. Endoplasmic reticulum stress triggers autophagy in malignant glioma cells undergoing cyclosporine a-induced cell death. Oncogene 32, 1518-1529 (2013).

21. Song, S., Tan, J., Miao, Y., Li, M. \& Zhang, Q. Crosstalk of autophagy and apoptosis: Involvement of the dual role of autophagy under ER stress. J. Cell. Physiol. 232, 2977-2984 (2017).

22. Lee, J. M. et al. Nutrient-sensing nuclear receptors coordinate autophagy. Nature 516, 112-115 (2014).

23. Ren, F. et al. Inhibition of glycogen synthase kinase 3beta promotes autophagy to protect mice from acute liver failure mediated by peroxisome proliferator-activated receptor alpha. Cell Death Dis. 7, e2151 (2016).

24. Lu, T. H. et al. Arsenic induces reactive oxygen species-caused neuronal cell apoptosis through JNK/ERK-mediated mitochondria-dependent and GRP 78/CHOP-regulated pathways. Toxicol. Lett. 224, 130-140 (2014).

25. Flister, K. F. T. et al. Long-term exposure to high-sucrose diet down-regulates hepatic endoplasmic reticulum-stress adaptive pathways and potentiates de novo lipogenesis in weaned male mice. J. Nutritional Biochem. 62, 155-166 (2018). 